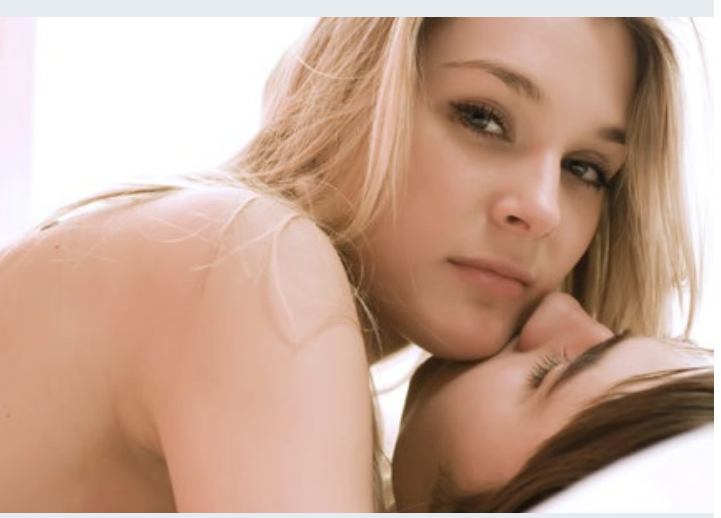

\section{Maßgeschneiderte Verhütung}

Wenn es um Verhütung geht, ist die individuelle Beratung in der frauenärztlichen Praxis unverzichtbar. Nicht nur vor dem Hintergrund zunehmender juristischer Auseinandersetzungen wird dabei die sorgfältige und dokumentierte Aufklärung der Patientin immer wichtiger.

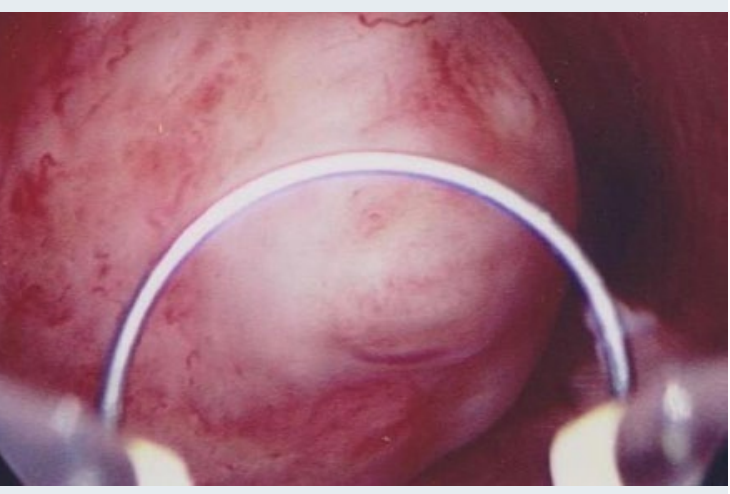

27 Therapieoptionen bei Myomen

Antworten auf die wichtigsten Fragen zum Thema Behandlung von Myomen und myombedingter Beschwerden gibt Prof. Matthias David im CME-Beitrag.

\section{Literatur kompakt}

8 Fehlbildungsrisiko durch die Pille?

9 Gentest kann Frauen mit Brustkrebs die Chemo ersparen

10 Vorzeitiger Blasensprung: Abwarten lohnt sich

\section{Fortbildung}

12 Die Vaginalflora-Diagnostik in Praxis und Labor - Teil 9 B-Streptokokken: gefährlich nur für das Neugeborene Eiko E. Petersen

16 Update Kontrazeption

Frauenärztliche Beratungskompetenz wichtiger denn je Elisabeth Merkle

21 Diagnostik und Therapie von Trophoblasterkrankungen Überarbeitete Leitlinie gibt Orientierung Clemens Tempfer

27 CME: Antworten auf die wichtigsten Fragen Aktuelles Behandlungsspektrum bei Myomen Matthias David

\section{Titel}

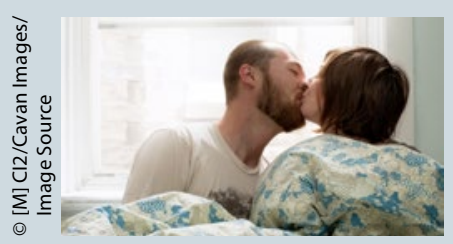

Titelthema dieser Ausgabe: Update zur Kontrazeption

\section{Schriftleitung}

Prof. Dr. med. Bernd Kleine-Gunk Schön Klinik Nürnberg/Fürth Europaallee 1, 90763 Fürth

E-Mail: kleine-gunk@euromed.de

Prof. Dr. med. Peter Mallmann Klinik und Poliklinik für Frauenheilkunde und Geburtshilfe, Universität zu Köln Kerpener Straße 34, 50931 Köln E-Mail: peter.mallmann@uk-koeln.de

\section{Rubriken}

3 Editorial

6 Panorama

53 Industrieforum

54 Impressum

55 Die letzte Seite 


\section{Medizin aktuell}

\section{FOKO 2016}

Wissenschaft und Berufspolitik in engem Zusammenspiel

36 32. Deutscher Krebskongress, 2016

Drehpunkt: individualisierte und altersgerechte Therapiekonzepte

38 Weiterentwicklung des Zervixkarzinom-Screenings

Wie wird die künftige Strategie aussehen?

40 Ergebnisse der Online-Umfrage

Work-Life-Balance: So finden Sie Ihr Gleichgewicht

\section{NATUM}

44 Gynäkologisch-onkologische Komplementärmedizin

Bromelain, ein multipotenter Bioregulator

\section{Geno Gyn}

\section{Bedeutung des intestinalen Mikrobioms \\ Gesundheit beginnt im Darm}

47 Hygiene in der Praxis: ein Fall für Profis

\section{Praxis konkret}

\section{Alles was Recht ist}

Keine Pflicht zum bedingungslosen Schwangerschaftsabbruch

49 Hamburger Initiative Babylotsen

Eine helfende Hand für junge Eltern

50 Die Zukunft der zertifizierten Fortbildung

Es steht ein Wandel in der Struktur und den Inhalten bevor

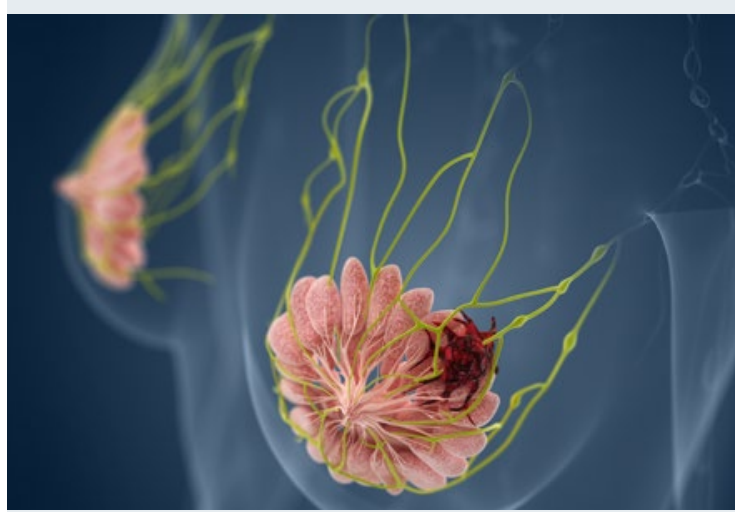

\section{Neues vom FOKO 2016}

Beim Fortbildungskongress (FOKO) stand wieder praxisnahe Wissensvermittlung im Fokus. Neben der Diagnostik und Therapie des Mammakarzinoms oder dem Klimakterium widmete sich ein Hauptthema auch rechtlichen Brennpunkten in der Gynäkologie.

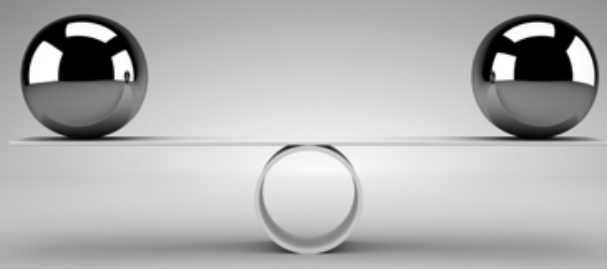

\section{Ihre Work-Life-Balance}

In unserer Umfrage wollten wir vor Kurzem erfahren, wie es um Ihr Verhältnis zwischen Arbeits- und Privatleben, um Ihre berufliche Belastung und um Ihre Zufriedenheit steht. Wir präsentieren Ihnen nun die Ergebnisse.

\section{Verlagsredaktion}

Dr. Claudia Mäck (Ressortleitung)

E-Mail: claudia.maeck@springer.com Tel.: 089/203043-1403

Springer Medizin Verlag GmbH

Aschauer Straße 30

81549 München

\section{Newsletter}

Update Gynäkologie

Alle 14 Tage das Neueste aus Forschung, Praxis und Berufspolitik bietet Ihnen der E-Mail-Newsletter Gynäkologie Update. Für diesen kostenlosen Dienst und für weitere Fach-Newsletter können Sie sich anmelden nach Registrierung auf:

www.springermedizin.de

\section{Organschaften}

GenoGyn - Ärztliche Genossenschaft für die Praxis und für medizinisch-technische Dienstleistungen e.G.

NATUM - Arbeitsgemeinschaft für Naturheilkunde, Komplementärmedizin, Akupunktur und Umweltmedizin in der Deutschen Gesellschaft für Gynäkologie und Geburtshilfe 